\title{
Magnetic resonance imaging of neonates in the magnetic resonance compatible incubator
}

\author{
Monika Bekiesińska-Figatowska1 ${ }^{1}$ Ewa Helwich², Magdalena Rutkowska², Joanna Stankiewicz³, \\ Iwona Terczyńska ${ }^{4}$
}

\begin{abstract}
${ }^{1}$ Department of Diagnostic Imaging, Institute of Mother and Child, Warsaw, Poland ${ }^{2} \mathrm{Clinic}$ of Neonatology and Neonatal Intensive Care, Institute of Mother and Child, Warsaw, Poland

${ }^{3}$ Intensive Care and Anesthesiology Clinic, Institute of Mother and Child, Warsaw, Poland

${ }^{4}$ Clinic of Neurology of Children and Adolescents, Institute of Mother and Child, Warsaw, Poland
\end{abstract}

Submitted: 14 April 2014

Accepted: 14 June 2014

Arch Med Sci 2016; 12, 5: 1064-1070

DOI: $10.5114 /$ aoms.2016.61913

Copyright $\odot 2016$ Termedia \& Banach

\section{Abstract}

Introduction: The authors present the first experience in neonatal magnetic resonance imaging (MRI) examinations using an MR compatible incubator (INC) at the Institute of Mother and Child.

Material and methods: Forty-nine examinations of 47 newborns (20 girls, 27 boys) were performed using the GE Signa HDxt 1.5T system and INC Nomag IC 1.5. Demographic data, anesthetic methods and MRI findings in the INC in comparison with previously performed imaging were analyzed.

Results: Thirty-two neonates were prematurely born $(68.1 \%)$ at gestational age 23-37 weeks, mean: 29.9 weeks. They were examined at 26 weeks postmenstrual age to 1 month corrected age, mean: 37.5 weeks. Body weight of newborns on the study day was 600-4300 g, mean: $2724 \mathrm{~g}$. Seventeen $(34.7 \%)$ children were examined in physiological sleep, $32(65.3 \%)$ anesthetized. In none of them did anesthesiological complications or disease worsening occur. In 43 (91.5\%) children brain MRI was performed, in 4 (8.5\%) MRI of the spinal cord and canal and of the abdomen/pelvis. In children prenatally examined by MRI, the INC provided new diagnostic information in $5(83.3 \%)$ cases, in neonates studied after birth by ultrasound in $32(82 \%)$. Magnetic resonance imaging in the INC did not entail additional knowledge in $9(18.7 \%)$ cases.

Conclusions: The INC enables MRI in preterm newborns and those with low/ extremely low body weight. These studies are necessary to assess the extent of changes in the central nervous system and other organs. Incubator coils, designed specifically for neonates, allow more accurate diagnosis than previously used coils for adults. MRI results allow one to determine prognosis, for more accurate planning of diagnostics, helping to make appropriate therapeutic decisions.

Key words: neonates, magnetic resonance imaging, MR compatible incubator (INC).

\section{Introduction}

Magnetic resonance imaging (MRI) studies of neonates have been performed for years (and still are performed) in specialized centers

\author{
Corresponding author: \\ Monika Bekiesińska- \\ Figatowska MD, PhD \\ Department of \\ Diagnostic Imaging \\ Institute of Mother and Child \\ 17 a Kasprzaka St \\ 01-211 Warsaw, Poland \\ Phone: +48 223277 156, \\ +48223277306 \\ Fax: +48 223277195 \\ E-mail:m.figatowska@mp.pl
}


equipped with high field MRI systems and experienced staff. Although it is highlighted in the literature and known from personal experience that one can examine safely even premature newborns without a dedicated MRI unit or MR compatible incubator $[1,2]$, the emergence of such equipment changes the situation, especially in terms of access to the study for the smallest and the most seriously sick premature infants [3]. Last year, two neonatal centers in Poland received the first two incubators compatible with MRI scanners (called the MR compatible incubator, INC). The first radiological only experience with one of these incubators at the Institute of Mother and Child (IMiD) in Warsaw has already been described [4], and after more research the authors present neonatal examinations in the INC from the viewpoint of not only a radiologist, but also a neonatologist, anesthesiologist and pediatric neurologist.

\section{Material and methods}

The material consists of 47 newborns examined at the Department of Diagnostic Imaging of IMiD (20 girls, 27 boys), including one child examined twice in order to assess the evolution of le- sions after cardiac arrest and one examined twice due to the failure of the initial study - a total of 49 studies were performed. Magnetic resonance examinations were performed using a $1.5 \mathrm{~T}$ scanner in the MR compatible incubator Nomag IC 1.5. The equipment of the INC is presented in Table I.

The studies were conducted in babies' physiological sleep or under general anesthesia. Atropine and thiopental in a dose of $4-7 \mathrm{mg} / \mathrm{kg}$ of body weight were used to induce anesthesia. In patients with preserved own breath, passive oxygen therapy in the INC chamber was used and anesthetized babies with inefficient breathing were ventilated with a respirator integrated within the INC. Vital parameters, heart rate and oxygen saturation were monitored using a wireless pulse oximeter adapted to work in the magnetic field. During the study - in the absence of infusion pumps dedicated to work in a magnetic field - the supply of intravenous fluids was withheld. The time period without infusion did not exceed 30 min.

We analyzed demographic data of the newborns, their anesthetic requirements and the results of MRI in INC in combination with the previously performed imaging studies: in 6 children

Table I. Parts of the incubator

\begin{tabular}{|cl|}
\hline No. & \multicolumn{1}{c|}{ Description } \\
\hline 1. & An infant incubator system suitable for MR imaging. Compatible with MR scanners up to 1.5 T. Suitable \\
& for scanning neonates with body weight up to $4.5 \mathrm{~kg}$ and maximum body length of $55 \mathrm{~cm}$ : \\
& - Air temperature and humidity regulation \\
\hline 2. & Trolley for intra-hospital transport, suitable for use with MR scanners of field strengths up to $1.5 \mathrm{~T}$ \\
& equipped with: \\
& - Shock absorbers \\
& - Four gas cylinders holders \\
& - UPS with supply capability up to $3 \mathrm{~h}$
\end{tabular}


with prenatal MRI study, in 37 cases with postnatal ultrasound (US); in 3 cases of children from other centers US reports were not delivered.

\section{Results}

In our study group there were 32 preterm newborns, which accounts for 68.1\% (32/47); they were born between 23 and 37 weeks of gestation, at the mean age of 29.9 gestational weeks. They were examined in the INC between 26 weeks of post-conception age and 1 month of corrected age, mean 37.5 weeks post-conception. The percentage of term newborns was $31.9 \%(15 / 47)$. At the day of the study the body weight of our patients was from $600 \mathrm{~g}$ to $4300 \mathrm{~g}$, mean: $2724 \mathrm{~g}$.

In 17 cases $(17 / 49=34.7 \%)$ the neonates were fed half an hour before the planned procedure, and these babies were examined in physiological sleep. In 32 cases $(32 / 49=65.3 \%)$ the newborns were anesthetized. Twenty-nine patients from this group $(29 / 32=90.1 \%)$ preserved their own breath. Three children from the group of generally anaesthetized $(3 / 32=9.4 \%$, including the most premature one of 26 weeks of postmenstrual age and $600 \mathrm{~g}$ of body weight), with inefficient breathing, required ventilation by means of a respirator integrated into the INC. All neonates that were subjected to anesthesia had efficient circulation and did not require the supply of catecholamines. None of the newborns studied in anesthesia showed any complications or worsening of the disease.

In 43 (91.5\%) of 47 neonates brain MRI was performed. Four babies $(4 / 47=8.5 \%)$ were referred for MRI of the spinal canal and cord and abdomen/ pelvis. In the group of babies prenatally examined by MRI, the INC examination provided new diagnostic information in 5 cases $(5 / 6=83.3 \%)$, in neonates studied after birth by ultrasound in 32 cases $(32 / 39=82 \%)$. Magnetic resonance imaging in the INC did not entail additional knowledge about the pathology in 9 cases $(9 / 48=18.7 \%)$.

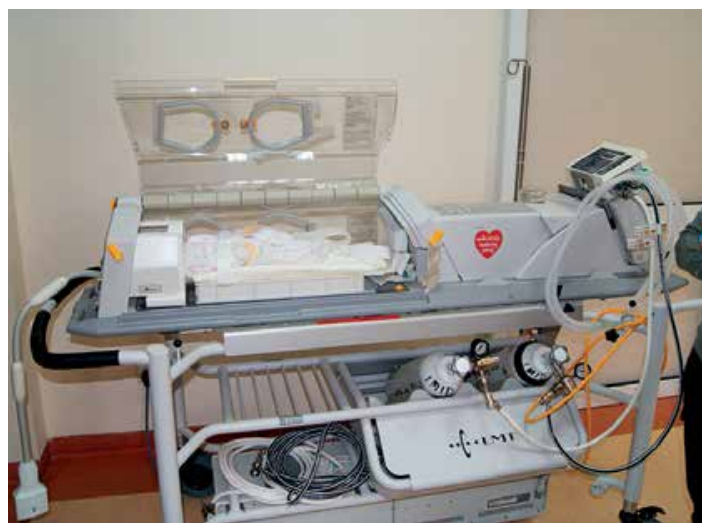

Figure 1. The MR compatible incubator

\section{Discussion}

There is no doubt that neuroimaging studies are necessary in those neonates, both preterm and term, in whom brain abnormalities are suspected: congenital malformations, perinatal injury, pathology typical of prematurity. There is also no doubt that transfontanel ultrasonography is and will remain the primary diagnostic method as a non-invasive, readily available and cheap examination. However, it has its limitations, and at the present time magnetic resonance imaging is the most accurate way to examine the brain and an essential tool for assessing changes within it and the prognosis for the development of the newborn baby [5].

Looking through the literature to find recommendations on neuroimaging in neonates, one can only find the American recommendations published in 2002 in Neurology [6]. This is a report of the Quality Standards Subcommittee of the American Academy of Neurology and the Practice Committee of the Child Neurology Society, divided into sections on imaging of children born prematurely and term neonates. As far as the first group is concerned, the authors admit that ultrasound and MRI performed on the same day in the first week of life differ significantly in terms of the amount of information provided, to the benefit of magnetic resonance imaging, which shows, above all, more white matter abnormalities, better depicts fine and more peripherally located hemorrhages and more accurately shows cystic changes in their spatial location. At the same time, however, they state that there is insufficient justification for routinely performing $M R I$ of the head in all newborns with very low body weight who have brain abnormalities on ultrasound. As for the second group of neonates, they divide it further into two subgroups: children with a history of neonatal encephalopathy, severe birth trauma, low hematocrit, and coagulopathy, and babies with acute encephalopathy. For the first subgroup they recommend a CT scan without contrast agent in search of bleeding, and, if $\mathrm{CT}$ does not explain the clinical condition of the newborn, MRI. For the second subgroup they recommend $\mathrm{MRI}$ between the $2^{\text {nd }}$ and $8^{\text {th }}$ day of life [6]. Twelve years have already passed since the publication of these recommendations, and technological progress has changed a lot in the field of MRI. With the current capabilities of MRI, the CT scan may be omitted in the diagnostic process in those centers which are equipped with an MRI scanner. Also, MR compatible incubators for newborns have been invented, and one of them is the subject of this work (Figure 1). It should be added here that Polish recommendations for neuroimaging of newborns are currently being developed in our center. 
It should also be noted that although brain abnormalities are the main indication to perform MRI in neonates, it is not the only one. In our material it is illustrated by the following figures: MR imaging of the brain was performed in $91.5 \%$ of cases, and in $8.5 \%$ it was used to evaluate the spine and spinal canal, abdomen and pelvis. Technical progress allowed the incorporation in incubators not only of the coils to study the brain, but also of the coils to study the whole body of the newborn. This is the second report on the studies not only of the brain in the world literature. Among the very few - seven - original works published since 2002 and devoted to neonatal MRI studies in the INC, the absolute majority discuss only brain imaging [3, 7-12]; one cannot even say neuroimaging studies, because the term "neuroimaging" refers also to the spinal cord, and this has hitherto been unavailable to studies in the INC. Only one publication describes - among 13 MRI studies - two cardiac and two pelvic examinations in the INC [13]. In our first case which did not concern the brain an unusual pathology was found in the form of myelomeningocele under the skin of a newborn, and additionally renal agenesis, ectopic kidney and unicornuate uterus. In the second case we excluded the relationship of the abdominal cyst to the biliary ducts and ovaries and established the most probable diagnosis of gastrointestinal duplication cyst; the differential diagnosis was mesenteric cyst (Figure 2). Intraoperatively duplication of the gastrointestinal tract was confirmed. In the third case the examination showed no connection between the visible and palpable seeping thickening of the skin in the coccygeal area and vertebral canal. Low position of the medullary cone was detected at the level of the $L 5$ vertebra with lack of hindbrain herniation. In the fourth case lipomyelomeningocele was detected in the sacral spine with a minimal fatty component in the spinal canal and a bigger one in the subcutaneous tissue with a tethered cord, without hindbrain herniation as well.

The INC is a response to the fear of performing MRI in preterm and term neonates who are in a serious condition, because it provides them the opportunity to conduct continuous intensive care and monitoring during a diagnostic procedure [14]. What is extremely important in the qualification of the newborns for MRI is the stability of their clinical condition. If the baby is unstable, then first of all one has to determine whether the information that MRI is expected to provide is critical for dealing with the neonate at this point of time. If the answer is "no", it should be postponed until the general condition of the newborn is improved [15].

Magnetic resonance imaging in an unstable clinical condition in premature neonates (when the infant requires a constant infusion of catecholamines due to severe hypotension which is difficult to correct) is performed very rarely, in order to establish the prognosis for survival when the sonographic picture of the brain is unclear. More accurate MRI assessment may have a significant impact on the further management. In term newborns it is easier to decide to perform MRI, even if their condition is unstable. If brain damage which is partially visible on ultrasound shows a wider range (e.g. bilateral and extensive periventricular venous infarcts), or if the effects of severe perinatal hypoxic-ischemic encephalopathy are not yet visible on sonography (and these may be extensive cortico-subcortical lesions and/or bilateral thalamic injury), their visualization by MRI is essential and can - in the presence of serious neurological symptoms - lead to important decisions as to further management because the prognosis for survival and development of the child is then serious. Magnetic resonance imaging of the brain allows detection of additional pathologies such as venous sinus thrombosis in which early treatment significantly improves the prognosis. In the standard US procedure detection of cerebral venous thrombosis is impossible; therefore it is very rarely recognized, and its prevalence is underestimated. In our study there were as many as 4 babies with thrombosis (4/47, i.e. $8.5 \%)$. It should be noted that venous sinus thrombosis may occur as an

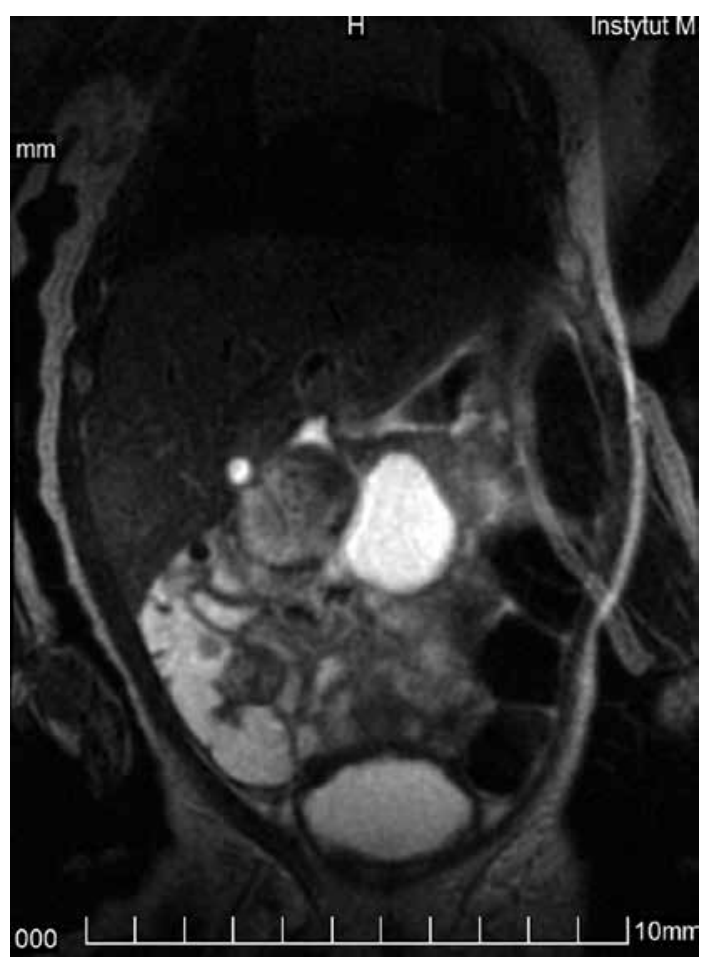

Figure 2. Term female neonate examined at the age of 11 days, body weight $4300 \mathrm{~g}$. The cystic mass in the midline does not show any relationship with the liver and biliary system or the ovaries 
isolated disease or coexist with hypoxic-ischemic changes or bleeding, which occurred in 3 of our patients. In the fourth case the acute extensive thrombosis worsened the already very bad in utero child's neurological condition resulting from the coexistence of brain malformation (pachygyria) and impaired myelination (Figure 3). The results of $M R I$ in this patient facilitated planning of metabolic and genetic diagnostics and allowed determination of prognosis and assessment of the chance of survival - in this case it was decided to implement palliative treatment only.

In the era of precision medicine detailed analysis of the initial central nervous system (CNS) structure and the overlapping pathology from both the prenatal and perinatal period is also a key to planning further diagnostics and therapy. Magnetic resonance examination of babies born as extremely immature (at the age of less than 28 weeks of gestation), which is performed at the age of about 40 weeks, allows the assessment of structures that are not visible on ultrasound (basal ganglia, internal capsule) or poorly visible (cerebellum). Magnetic resonance imaging studies are increasingly often performed in neonates, and their results indicate higher incidence of bleeding in the infratentorial compartment than previously believed. This is due to the fact that the posterior
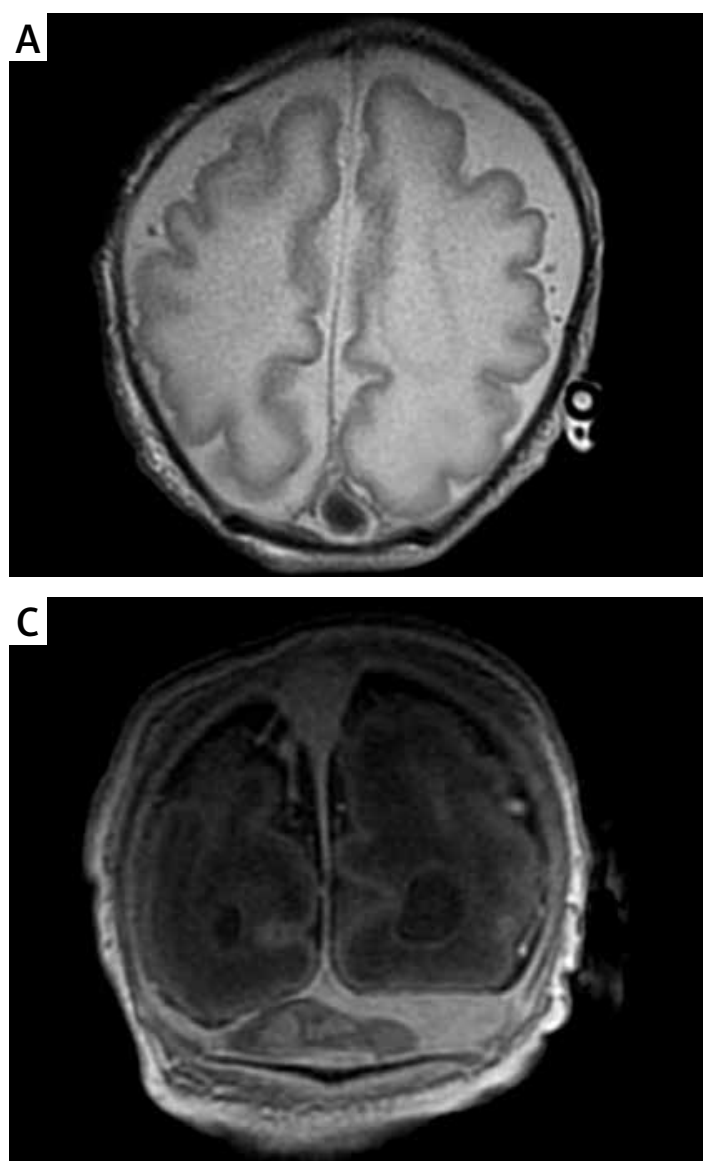

fossa pathology, including congenital anomalies, is more difficult to evaluate on US than lesions in the supratentorial compartment [16]. In addition, MR examination allows assessment of brain volume and the degree of white matter myelination. All this is important in predicting the further development of the child, planning specialist care and early intervention. Attention is now paid to the fact that cerebellar abnormalities (such as hemorrhage or hypoplasia) may have a future impact on the development of autism, which occurs in this group of infants 8-10 times more often than in the population of infants born at term $[17,18]$. In the analyzed material posthemorrhagic changes in the cerebellum and/or around it were found in nearly one-third of the neonates $(14 / 47=29.8 \%)$, including $31.2 \%$ of preterm babies (10/32), but also in $26.7 \%$ of term newborns (4/15). In one neonate $(1 / 47=2.1 \%) \mathrm{MRI}$ showed a congenital cerebellar anomaly: rhombencephalosynapsis (lack of cerebellar vermis, fusion of the hemispheres, dentate nuclei and superior cerebellar peduncles) (Figure 4). Additionally, posthypoxic-postischemic changes were found in the brain stem of one newborn after cardiac arrest.

However, not all of the changes seen in MR images within the CNS have prognostic significance. Magnetic resonance imaging studies performed in

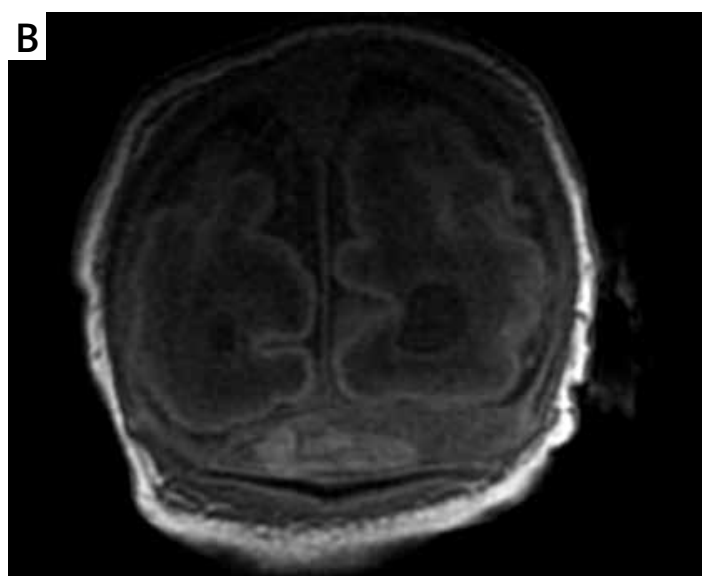

Figure 3. Term male neonate examined at the age of 6 days, body weight 2600 g. Pathological gyration (pachygyria) and abnormally myelinated white matter of the brain. Note the superior sagittal sinus abnormality (A). The thin slices show the thrombus in the transverse sinuses: brighter in comparison with the blood before the administration of contrast medium (B) and darker (without contrast enhancement) after its administration (C) 
extremely immature neonates and their (short for now) clinical evaluation indicate that - for example - severe, diffuse hyperintensity of the white matter (called DEHSI - diffuse excessive high signal intensity) is a transient phenomenon in this group of neonates $[19,20]$.

The above considerations are only one aspect of the method of MRI structural studies. But one has to keep in mind that MRI is not only a structural method; it is also a molecular imaging method, an alternative to positron emission tomography (PET), which is becoming more and more important in neuroscience and clinical neurology. With use of these MR applications one can study neuroplasticity of the developing brain, which - as it has already been shown - has the ability to be shaped by environmental inputs both under normal conditions and after an injury [21]. Diffusion-weighted imaging, MR spectroscopy, functional MRI, optical imaging, and specific targeted contrast agents are all used in molecular imaging, and some of these possibilities are also offered by the MR compatible incubator and may be used in the future, as this paper is an initial report only, after the first few months of the INC's use in our practice.

A detailed assessment of the whole brain in the INC is facilitated by the head coil, which is specially designed for neonates and can attain images of a much higher quality than the previously used coil for adults - at our center it was a head coil for the adults which housed the entire body of the child. The rest of the neonatal body was also studied in this coil when necessary, and now there are two additional special coils dedicated to body imaging. Together with distinct sequence protocols, designed for neonatal studies, with appropriately modified parameters, these coils allow for improved image quality [7] while scanning premature babies and sick infants up to a body weight of $4.5 \mathrm{~kg}$ and a maximum body length of $55 \mathrm{~cm}$. It is

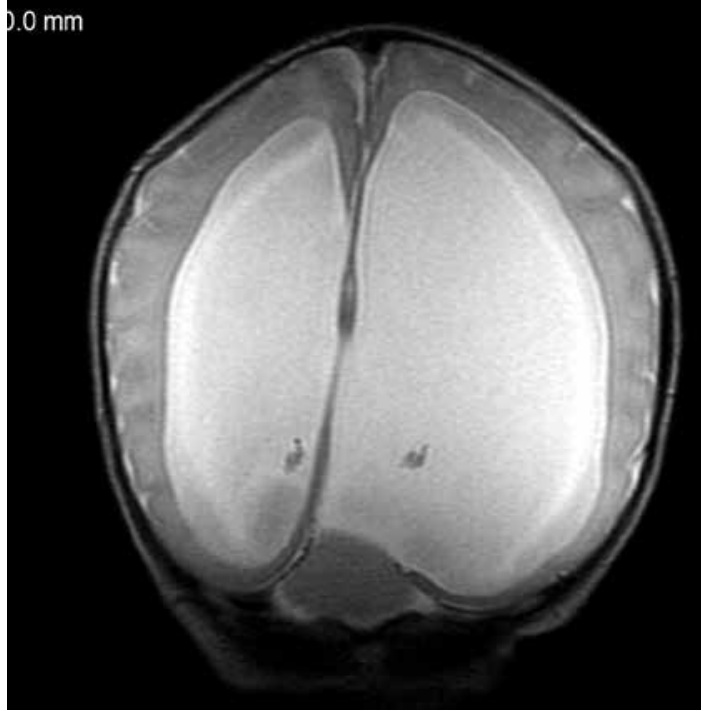

Figure 4. Preterm male neonate, body weight $2600 \mathrm{~g}$. Hydrocephalus, a small cerebellum without the vermis and no division into hemispheres - the cerebellar folia run continuously from one side to the other: rhombencephalosynapsis

also important that the INC allows more effective immobilization of the body and the head of the newborn than in the "adult" coil.

Not all newborns require pharmacological sedation to undergo MRI. Feeding the babies half an hour before the scheduled procedure allowed in just over one third of cases for them to get a physiological peaceful sleep and for the study to be performed during sleep. Comfortable conditions inside the incubator (optimum temperature and humidity) favored sleep as well. The patient's isolation from noise, which prevails in the MRI chamber, is another advantage of the INC.

Thanks to the advantages of INC, discussed above, we could safely perform MRI in our youngest premature baby at the age of 26 postmenstru-
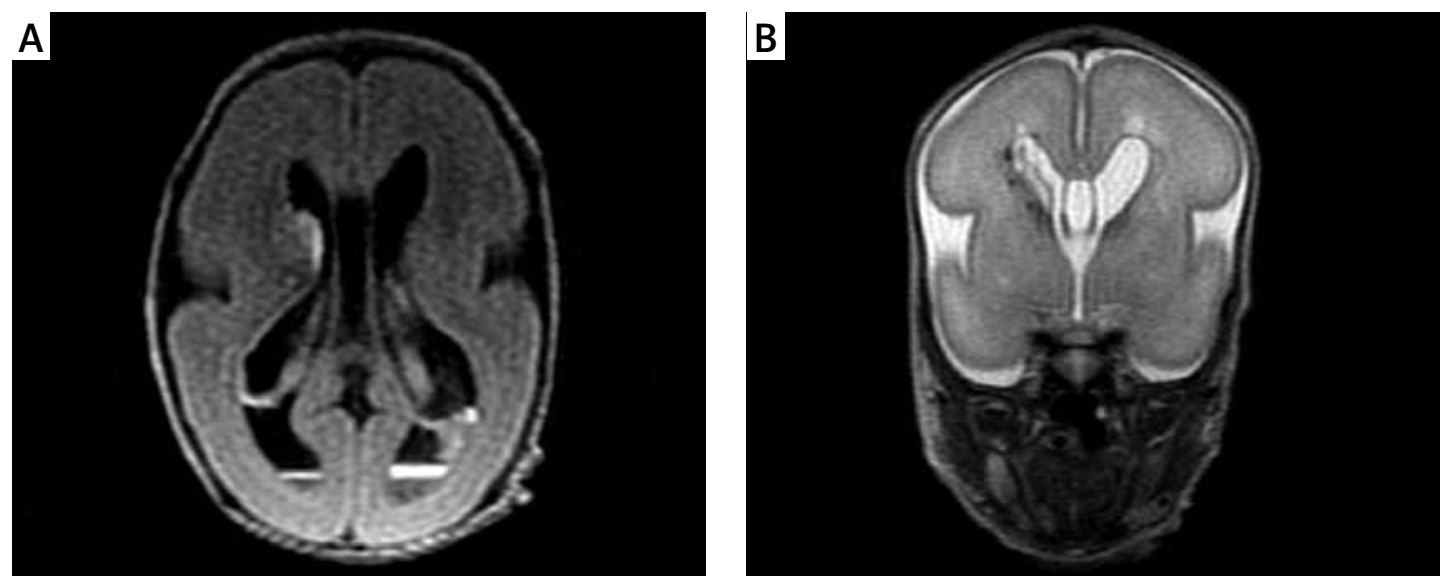

Figure 5. Preterm male neonate, born at the age of 23 gestational weeks, examined at the age of 26 weeks, body weight $600 \mathrm{~g}$. Hemorrhage into the germinal matrix/brain tissue on the right, subependymal and intraventricular hemorrhage, bleeding into the choroid plexi (A). Cavities over both lateral ventricles (B) 
al weeks, with the body weight of $600 \mathrm{~g}$. In this case in several US examinations during the first 2 weeks of life the findings were so equivocal that it was decided to perform MRI at such an early stage (Figure 5). To our knowledge, this is the smallest baby examined so far - in those published papers in which the body weights of individual patients are given, we found data that so far the smallest babies weighed $1200 \mathrm{~g}$ [13] and $1400 \mathrm{~g}$ [22].

Of course, special care is needed to maintain the purity of the incubator and appropriate disinfection after each study so that this additional equipment, in which the newborn is placed, does not become a source of infection [23].

In conclusion, the MR compatible incubator enables MRI studies in infants born prematurely and with low and extremely low body weight. These examinations are necessary to establish the diagnosis and to assess the actual extent of changes both in the central nervous system and in the other organs and systems. The coils designed specifically for newborns, which are an integral part of the incubator and are meant not only to study the brain, but also the whole body, allow more accurate diagnoses than previously used coils for adults. The results of MRI studies performed using this highly specialized equipment make it possible not only to determine the outcome, but also to plan precisely further diagnostics and help to make appropriate therapeutic decisions.

\section{Conflict of interest}

The authors declare no conflict of interest.

\section{References}

1. Benavente-Fernández I, Lubián-López PS, Zuazo-Ojeda MA, Jiménez-Gómez G, Lechuga-Sancho AM. Safety of magnetic resonance imaging in preterm infants. Acta Paediatr 2010; 99: 850-3.

2. Bekiesińska-Figatowska M, Borszewska-Kornacka MK, Antczak-Marach D, Szczepanik E. Regression of cystic lesions on brain MRI in a child with hypoxic-ischemic encephalopathy treated with selective head cooling [Polish]. Ginekol Pol 2013; 84: 151-6.

3. Rona Z, Klebermass K, Cardona F, et al. Comparison of neonatal MRI examinations with and without an MR-compatible incubator: advantages in examination feasibility and clinical decision-making. Eur J Paediatr Neurol 2010; 14: 410-7.

4. Bekiesińska-Figatowska M, Szkudlińska-Pawlak S, Romaniuk-Doroszewska $A$, et al. First experience with neonatal examinations with use of the MR compatible incubator. Pol J Radiol 2014; 79: 268-74.

5. Zupan-Simunek V, Rutkowska M, Bekiesińska-Figatowska M. Predictive value of magnetic resonance imaging (MRI) in cases of acquired brain injury in neonates [Polish]. Med Wieku Rozwoj 2011; 15: 385-93.

6. Ment LR, Bada HS, Barnes P, et al. Practice parameter: neuroimaging of the neonate: report of the Quality Standards Subcommittee of the American Academy of
Neurology and the Practice Committee of the Child Neurology Society. Neurology 2002; 58: 1726-38.

7. O'Regan K, Filan P, Pandit N, Maher M, Fanning N. Image quality associated with the use of an MR-compatible incubator in neonatal neuroimaging. Br J Radiol 2012; 85: 363-7.

8. Paley MN, Hart AR, Lait M, Griffiths PD. An MR-compatible neonatal incubator. Br J Radiol 2012; 85: 952-8.

9. Berman JI, Mukherjee P, Partridge SC, et al. Quantitative diffusion tensor MRI fiber tractography of sensorimotor white matter development in premature infants. Neuroimage 2005; 27: 862-71.

10. Deipolyi AR, Mukherjee P, Gill K, et al. Comparing microstructural and macrostructural development of the cerebral cortex in premature newborns: diffusion tensor imaging versus cortical gyration. Neuroimage 2005; 27 : 579-86.

11. Partridge SC, Mukherjee P, Henry RG, et al. Diffusion tensor imaging: serial quantitation of white matter tract maturity in premature newborns. Neuroimage 2004; 22: 1302-14.

12. Whitby EH, Griffiths PD, Lonneker-Lammers T, et al. Ultrafast magnetic resonance imaging of the neonate in a magnetic resonance-compatible incubator with a built-in coil. Pediatrics 2004; 113: e150-2.

13. Blüml S, Friedlich P, Erberich S, Wood JC, Seri I, Nelson MD Jr. MR imaging of newborns by using an MR-compatible incubator with integrated radiofrequency coils: initial experience. Radiology 2004; 231: 594-601.

14. Panigrahy A, Borzage $M$, Blüml S. Basic principles and concepts underlying recent advances in magnetic resonance imaging of the developing brain. Semin Perinatol 2010; 34: 3-19.

15. Mathur AM, Neil JJ, McKinstry RC, Inder TE. Transport, monitoring, and successful brain MR imaging in unsedated neonates. Pediatr Radiol 2008; 38: 260-4.

16. Steggerda SJ, Leijser LM, Wiggers-de Bruïne FT, van der Grond J, Walther FJ, van Wezel-Meijler G. Cerebellar injury in preterm infants: incidence and findings on US and MR images. Radiology 2009; 252: 190-9.

17. Limperopulos C. Autism spectrum disorders in survivors of extreme prematurity. Clin Perinatol 2009; 36: 791805.

18. Johnson S, Hollis C, Kochhar P, Hennessy E, Wolke D, Marlow N. Autism spectrum disorders in extremely preterm children. J Pediatr 2010; 156: 525-31.

19. De Bruine FT, van den Berg-Huysmans AA, Leijser LM, et al. Clinical implications of MR imaging findings in the white matter in very preterm infants: a 2-year follow-up study. Radiology 2011; 261: 899-906.

20. Kidokaro H, Anderson PJ, Doyle LW, Neil JJ, Inder TE. High signal intensity on T2-weighted MR imaging at term-equivalent age in preterm infants does not predict 2-year neurodevelopmental outcomes. Am J Neuroradiol 2011; 32: 2005-10.

21. Schaller B, Cornelius JF, Sandu N. Molecular medicine successes in neuroscience. Mol Med 2008; 14: 361-4.

22. O'Regan K, Filan P, Pandit N, Maher M, Fanning N. Image quality associated with the use of an MR-compatible incubator in neonatal neuroimaging. Br J Radiol 2012; 85: 363-7.

23. Sadowska-Krawczenko I, Jankowska A, Kurylak A. Healthcare-associated infections in a neonatal intensive care unit. Arch Med Sci 2012; 8: 854-8. 